\title{
perifèria
}

Número 7, Diciembre 2007

www.periferia.name

\section{Conocimientos acerca de plantas en la nueva ruralidad. Cambio social y agro ecología en el Parque Natural de Montesinho (Portugal)}

Amélia Frazão-Moreira - CRIA, FCSH - Universidade Nova de Lisboa ${ }^{1}$ Ana Maria Carvalho - Dept. Biologia - Escola Superior Agrária de Bragança ${ }^{2}$ Maria Elisabete Martins - Centro de Estudos de Antropologia Social, Lisboa ${ }^{3}$

\section{Resumen}

En las últimas décadas, las regiones montañosas de Portugal han sufrido transformaciones económicas y una intensa disminución de la población que conllevaran modificaciones visibles de los agroecosistemas y en consecuencia del paisaje. Un estudio de caso, conducido en un espacio natural protegido de la provincia portuguesa de Trás-os-Montes (2006-2007) - el Parque Natural de Montesinho - y del ámbito del proyecto de investigación "Etnobotânica do Nordeste Português: saberes, plantas e usos", pretende comprobar que los conocimientos sobre plantas, sus usos y correspondiente manejo son la expresión de cambios culturales inherentes a estas dinámicas sociales y ecológicas.

En un contexto social enmarcado por una "nueva ruralidad" (es decir, un modo diferente de encarar y vivir el mundo rural), coexisten conocimientos y usos tradicionales de plantas, muchos de raíz ancestral, y saberes adquiridos más recientemente e incorporados en la cultura local. El análisis de los conocimientos por género, edad, grado de escolaridad, experiencia migratoria de los informantes, permite enfocar y evaluar la pérdida de la memoria social y cultural.

Los resultados que se presentan remiten aún para la discusión conceptual de los conocimientos ecológicos y tradicionales. Además, conllevan a una reflexión acerca de la preservación de estos saberes/prácticas y de la conservación de la naturaleza en el contexto de espacios naturales protegidos.

Palabras-Clave: saber tradicional; uso de plantas; cambio social; ruralidad; Portugal.

1 Enviar correspondencia a: Amélia Frazão Moreira, amoreira@fcsh.unl.pt. CEAS/CRIA (Centro de Estudos de Antropologia Social/Centro em Rede de Investigação em Antropologia). Dpto de Antropologia, FCSH, Universidade Nova de Lisboa, Av. Berna, 26-C, 1069-061 Lisboa, Portugal.

2 Ana Maria Carvalho - CIMO (Centro de Investigação de Montanha). Dpto. de Biologia, Escola Superior Agrária. Campus de Santa Apolónia. 5301-855 Bragança. Portugal. anacarvpb.pt

3 Maria Elisabete Martins - CEAS/CRIA (Centro de Estudos de Antropologia Social/Centro em Rede de Investigação em Antropologia). Becaria del Projecto $\mathrm{POCl}$, "Etnobotânica do Nordeste Português: saberes, plantas e usos". betamartins@iol.pt 


\title{
perifèria
}

Número 7, Diciembre 2007

www. periferia.name

\begin{abstract}
For decades, the Northeastern Portugal, a mountainous territory along the Spanish border, has suffered from critical economic changes and a slow steady decline of the population that have had a degrading effect on the agroecosystems and rural landscape. A study case has been carried out inside a protected area from Trás-osMontes (2006-2007), the Natural Park of Montesinho supported by the research project "Ethnobotany of the Northeastern Region of Portugal: local knowledge, plants and uses". How these changes are affecting the system of local knowledge of plant resources and the maintenance of traditional plant use practices are some of the questions that we address.
\end{abstract}

Within a new social context, different rural life-ways have been designed. Traditional ecological knowledge (TEK) coexists with modern trends in plants knowledge and use, recently introduced in local culture by present-day media and popular books, for instance. People's knowledge, local perceptions and classifications of the natural environment have been confirmed and compared by gender, age, educational background, occupation and other personal data in order to analyse erosion of TEK and partial or total loss of social and cultural memory.

Collected data focus on a conceptual discussion of TEK and provide some clues to the understanding of how important are TEK preservation, and the careful management of the environment and of natural resources for the conservation status of protected areas.

Keywords: TEK; plant use; social changes; rural context; Portugal.

\section{I ntroducción}

Quintanilha es un pequeño pueblo de la frontera del nordeste interior de Portugal con la provincia española de Zamora. Comparte con sus vecinos castellanos (de Nuez y San Martín del Pedroso) el rio Maçãs, muchas memorias y usos ancestrales, pero también la costumbre reciente de pasear al atardecer por el entorno del pueblo.

Mucha información etnobotánica recogida en el ámbito del proyecto de investigación "Etnobotânica do Nordeste Português: saberes, plantas e usos" ${ }^{4}$ ha sido obtenida durantes estos paseos por el campo, por ejemplo, mientras caminábamos al lado de D. Amalia ${ }^{5}$, una vecina muy considerada por sus conocimientos acerca de plantas y usos.

\footnotetext{
4 «Proyecto financiado por la Fundação Ciência e Tecnologia (Portugal) - POCl/ANT/59395/2004.

${ }^{5}$ Los nombres de los informantes son ficticios.
} 


\section{perifèria}

Número 7, Diciembre 2007

www. periferia. name

En ciertas ocasiones no hemos podido tomar datos de un solo individuo porque se suele pasear en grupo, con amigos o familiares. También porque los paseantes se encuentran a menudo, debido a que los escasos itinerarios disponibles se cruzan a cada paso. Estos hechos propiciaron discusiones sobre nombres populares, morfologías o usos.

\section{Contexto y proyecto}

El pueblo de Quintanilha se localiza en la región fronteriza de la provincia portuguesa de Trás-os-Montes y su término está incluido en un espacio natural protegido, el Parque Natural de Montesinho (PNM). Al contrario de muchos otros pueblos del parque, Quintanilha está conectada a Bragança, la ciudad más cercana, por los $20 \mathrm{~km}$ de una vía rápida que tardan cerca de quince minutos a recorrer.

Parte de sus casi 90 casas ocupadas son la residencia permanente de personas mayores (jubilados o que se encargan de los trabajos agrícolas) y de agregados familiares, cuyos miembros (todos o al menos algunos) trabajan en actividades comerciales o en los servicios administrativos, en la ciudad de Bragança. Una decena de estas casas sólo está habitada durante el fin de semana, los días festivos o en el verano. Pertenecen a familias que residen habitualmente en Bragança, en ciudades más lejanas (Lisboa, Porto, por ejemplo) o que son emigrantes.

Los profundos cambios sociales y económicos acorridos después de los años sesenta del siglo $X X$, sobre todo relacionados con los flujos migratorios para el exterior y el éxodo rural hasta las ciudades de la región o del litoral, condujeron a un vaciamiento demográfico de los pueblos del PNM. Estos cambios, además de otros que se sucedieran en la sociedad portuguesa, se reflexionaron en la estructura económica y social de toda la zona (Baptista 1993; Brito et al 1996; Godinho 2006; Rodrigues 1998 y 2000).

Precisamente debido a este contexto, Quintanilha se presenta entre un pasado muy liado con la actividad agropecuaria y un futuro en que el pueblo se antevé como un "dormitorio" de Bragança. El pueblo vive entre una perspectiva campesina del mundo (Pina-Cabral 1986; Pinto 1985) que ha enmarcado su pasado y una "nueva ruralidad" que se puede traducir como un otro modo de vivir el campo, cruzando 


\section{perifèria}

Número 7, Diciembre 2007

www.periferia. name

valores y representaciones sociales de génesis campesina con expectativas y comportamientos urbanos y burgueses ${ }^{6}$.

En el entorno del pueblo, los sistemas agrarios han cambiado y los agroecosistemas locales, que son producto de la intervención humana durante siglos, consustanciada en la diversidad de actividades agropecuarias, en el aprovechamiento de los recursos disponibles y en las formas de usar el territorio y ocupar los suelos (Carvalho 2005; ESAB/PNM 2007; O’Neill 1984; Rodrigues 1998 y 2000), sufren profundas transformaciones a lo largo de las últimas dos décadas.

Algunos ejemplos nos remiten para estos hechos: (i) las tradicionales "faceiras" (parcelas de campo abierto donde se cultivaban cereales en rotación bienal) han sido invadidas por un flora arbustiva y herbácea. Dan ahora lugar a matorrales, piornales brézales o jarales; (ii) la vegetación propia de los pastizales típicos o "lameiros", que se caracterizaba por su gran diversidad y rusticidad, se está perdiendo porque el manejo de estos pastos ya no se hace como antiguamente (la hierba es segada menos veces o no hay ganado para pastorear); (iii) la gente, a quienes aún les importa mantener sus tierras productivas, prefiere optar por los cultivos arbóreos de crecimiento rápido, incluso especies de desarrollo un poco más lento, desde que no exijan una atención diaria y proporcionen un rendimiento económico apreciable, como sean las choperas (Populus spp.), los cerezales (Prunus avium.) o los castañares (Castanea sativa); (iv) los huertos familiares son muchas veces sustituidos por jardines, donde se cultivan, fundamentalmente, especies exóticas ornamentales, que son consideradas como un signo de prosperidad y modernidad.

\footnotetext{
${ }^{6}$ En este contexto entendemos "ruralidad" como una realidad social marcada por un tipo diferente de organización y racionalidad económicas y un modo de vida relativamente autónomo. Estamos conscientes que importa discutir el significado actual de los conceptos de "rural" y "urbano". Sin embargo, esa reflexión non es el objetivo de esta comunicación.
} 


\section{perifèria}

Número 7, Diciembre 2007

www. periferia.name

Esta dinámica de cambios sociales, económicos y agroecológicos, también observable en otros pueblos de la zona, pero menos intensamente, nos ha llevado a elegir Quintanilha como el universo de este primero estudio de caso del ámbito del proyecto "Etnobotânica do Nordeste Português: saberes, plantas e usos".

Dicho proyecto tiene como objetivo comprender cómo se procesa y actualiza la apropiación social del universo vegetal en contextos rurales y en tiempos de cambio social y económico. Pretendemos aprehender la sabiduría relativa a los recursos vegetales, considerando su componente utilitaria, identificar y describir prácticas sociales actuales, en las cuales se recurre al uso de plantas y recoger conocimientos y modos de hacer que quedan tan solo en la memoria de las personas, utilizando para el efecto metodología etnográfica y entrevistas etnobotánicas.

A la vez, queremos aclarar cómo se conciben y clasifican localmente las plantas y cómo dichas concepciones son manipuladas por los diferentes grupos de actores sociales, definidos teniendo en cuenta el género, edad, aptitudes y experiencia individuales. Con ese objetivo hemos realizado lo que hemos llamado "entrevistas con plantas" a que nos referiremos adelante.

En estos momentos, hemos concluido el trabajo de campo del primero estudio de caso, que respecta a Quintanilha, y se ha iniciado ya el proceso de análisis de la información, por lo cual los datos que ahora se presentan deben de ser entendidos como un acercamiento preliminar a los resultados.

\section{Memoria de usos y nuevos saberes}

Los saberes sobre las plantas que hemos encontrado y sus usos, así como las disputas de D. Amalia, de que hicimos eco en el introito, nos revelan que hay diferentes "mundos", es decir diferentes modos de conceptualizar y encarar el universo vegetal. A partir de información y de material vegetal proporcionados por los informantes hemos determinado cerca de dos centenares de plantas utilitarias y registrado más de una categoría de uso para muchas de ellas. 


\section{perifèria}

Número 7, Diciembre 2007

\section{www.periferia.name}

La memoria pretérita de los usos de plantas es enorme. La gente recuerda las plantas con usos artesanales, como las que utilizaban en cestería - "vimes, salgueiras, escovas, gesta piorneira" (mimbres, sauces, escobas, piorno) - o para fabricar zuecos como el "amieiro" (aliso, Alnus glutinosa); las plantas combustibles, "lentisco" (Phyllyrea angustifolia), "urze roxa" (Erica australis); las alimentarias, "azedas" (Rumex spp.), "rabaça" (Rumex obtusifolius) y "conecos" (Silene vulgaris), que se comían en ensaladas, además de muchos otros ejemplos, incluyendo las aplicaciones medicinales de la mayoría de las especies referidas por los informantes.

Algunos de estos usos aún están vigentes. Entre ellos el aprovechamiento de la "carqueja" (Pterospartum tridentatum subsp. tridentatum) para encender la lumbre, del "carrasco" (Quercus ilex subsp. ballota) para leña de calidad insuperable, o de la "escova-amarela" (Cytisus scoparius) de cuyas ramas se elaboran pequeñas escobas para barrer terrizos, patios y entradas. En la categoría de condimentarias siguen siendo insustituibles, "cheirosinha (Thymus zygis) para curar y aliñar las aceitunas y "arçã o arcenha" (Lavandula stoechas) adobo de caza o de conejo doméstico para que su gusto se parezca al del conejo salvaje.

Los conocimientos y usos relativos a plantas medicinales son aquellos que más perduran, como comprueba la recomendación y el consumo frecuente de infusiones medicinales de "cheirosinha", de flores del "caneleiro" (Sambucus nigra) y de "carqueja", de bayas de jara, "esteva" (Cistus ladanifer), para el catarro y la tos, además de los cocimientos de "borragem" (Borago officinalis) que regula el funcionamiento de los intestinos o de hierba "alcária" (Xolantha tuberaria) que apacigua las inflamaciones y dolencias del aparato urinario.

Sin embargo, sobre todo en lo que respecta a la categoría de plantas medicinales, es difícil discernir los conocimientos etnobotánicos de raíz ancestral, trasmitidos oralmente a lo largo de generaciones, de los recién adquiridos por medio de la 


\section{perifèria}

Número 7, Diciembre 2007

www. periferia.name

lectura directa de libros $u$ otras publicaciones de carácter divulgativo ${ }^{7}$, de programas de televisión, fundamentalmente emitidos por las cadenas españolas, como por ejemplo "Saber vivir" y "La botica de la abuela", pero también por intercambio de información con amigos, familiares o, incluso, con algún experto en medicinas convencionales y/o terapias alternativas.

Las memorias del trabajo y de los juegos de la infancia con recurso a las plantas se conjugan con las certezas científicas aprendidas recientemente por otros medios.

A lo largo de una misma conversación el agricultor Dionisio (63 años de edad, 2oㅡ año del segundo ciclo de la enseñanza obligatoria) transita, frecuentemente, entre los relatos nostálgicos de sus juegos de niñez y la importancia de conocer la composición química de las especies medicinales de la región. Nos cuenta como los niños elaboraban pequeñas cestitas de "peliços", las cabezuelas de una compuesta (Arctium minus), para regalar a su profesora de primaria, y como, bastante más tarde, debido a sus parcos recursos económicos, en los domingos se perfumaba con agua de macerar las flores de violetas (Viola spp.) en la que mezclaba también un poco de jabón. Nos enseña sus artes de pescador y como preparaba los cebos para pescar con la raíz tóxica del "rabação" (Apium nodiflorum), previamente machacada y amasada con tierra. Recuerda también la multitud de alimentos silvestres que los niños conseguían en el campo, mientras jugaban o pastoreaban el ganado y nos describe la consistencia y el gusto exquisito de "chupetas" (flores de Trifolium pratense), "fogaças" (frutos de Malva sylvestris), "carneixos o corniçós" (vainas de Astragalus cymbaecarpos), "botas" (agallas de los talos de Hipochoeris glabra), "canastras" (vainas de Astragallus pelecinus), "riqueijões" (tallo subterráneo de Conopodium majus), "azedas y azedões" (hojas y talos tiernos de Rumex spp.), "moras y abrunhos" (frutos de Rubus spp. y de Prunus spinosa). De repente, se detiene cerca de un bueno ejemplar de "gilbardeira" (Ruscus aculeatus) y nos

\footnotetext{
${ }^{7}$ Nos han enseñado, por ejemplo, las siguientes obras: Boavida, M. F. (coord.) (s/d), As plantas nossas amigas - A saúde pelas plantas. 10 volumes. Lisboa: Amigos do Livro Editores; Pamplona, R. (1997) A saúde pelas plantas medicinais, Enciclopédia de Educação e saúde. 2 volumes, Sacavém: Publicações Atlântico; Pinto, A. (1999). O Boticário. Moderna Editorial Lavores.
} 


\section{perifèria}

Número 7, Diciembre 2007

www. periferia. name

explica que ha leído hace poco en un libro que la carne de los frutitos de esta planta es muy rica en calcio.

I gual que Dionisio otros varones y mujeres de la misma franja de edad (50/65 años) están ávidos de nuevos conocimientos acerca de la flora local y aspiran por informaciones eruditas que enriquezcan su sabiduría. Por ello, son lectores atentos de los libros que encuentran sobre el tema y son buenos oyentes de aquellos que les aportan más elementos relativos a las plantas. Aprehenden un cierto tipo de conocimiento que, deliberadamente, no clasificamos de "letrado" o "científico" porque, en la mayoría de las veces, se trata únicamente de una sistematización de un saber popular exógeno transmitido ahora por medio de la escritura.

Varones y mujeres, mientras crecían, interiorizaron y manipularon un cuerpo de conocimientos y practicas sobre plantas, construido en el seno de un sistema de supervivencia muy dependiente de los recursos naturales disponibles. Hoy día, fácilmente adhieren a los productos naturales y abrazan la idea de una naturaleza encarada como un valor a defender y proteger, siguiendo, eventualmente, las nuevas tendencias de las sociedades europeas (Milton 1993).

Según esta perspectiva, es defendible que los saberes exógenos terminarán por incorporarse en la lógica y cuerpo de los conocimientos locales, como Ellen (2000:28) preconcibe para el conocimiento indígena:

It will always have to be translated and adapted to local situations and will still depend on what individuals know and reconfigure culturally independently of formal and book knowledge.

Las plantas han dejado de ser manejadas como recursos utilitarios y silenciosos de un sistema agrario, para, mediante un cambio de circunstancias sociales y culturales, pasaren a ser entendidas como valores y los conocimientos con ellas relacionados enaltecidos y verbalizados. 


\section{perifèria}

Número 7, Diciembre 2007

www.periferia. name

\section{Diferentes edades - Diferentes conocimientos}

La segunda forma utilizada para recoger información etnobotánica fueron las ya referidas "entrevistas con plantas". Dichas entrevistas han ido realizadas a 42 sujetos de ambos sexos, con edades que variaron entre 10 y 84 años, con distintos niveles de escolarización y diversas experiencias de vida. Les presentamos 11 plantas $^{8}$, elegidas de entre las que han sido mencionadas durante la primera etapa de recolección de datos y hemos solicitado a cada uno, individualmente, que nos indicara el nombre de la planta y que nos contara todo lo que sabía acerca de cada una ${ }^{9}$.

Los datos obtenidos están siendo tratados estadísticamente pero los resultados de estos análisis aún no están disponibles. Sin embargo, usando algunos ejemplos ya es posible inferir alguna diferencia de conocimiento entre los informantes.

Todos, con excepción de un niño, han reconocido y nombrado la "carqueja". En cuanto a sus usos, hemos observado que es unánime el conocimiento de su aplicación como planta combustible mientras sus virtudes medicinales fueron mencionadas y descritas por 32 individuos, tan solo adultos y personas mayores, sin ningún joven que lo hiciera.

A su vez, "erva-prata" (Paronychia argentea), una sencilla herbácea con propiedades farmacológicas, solo ha sido determinada por 10 de los sujetos, todos con edades superiores a 55 años. Catorce personas han reconocido que se trataba de una planta medicinal, los 10 que la han nombrado y otros 4 individuos (de estos solo uno es joven) que no pudieron recordar o no sabían su nombre.

\footnotetext{
8 Las once plantas fueron: "arçã" (Lavandula stoechas), "agrião" (Rorippa-nasturtium- aquaticum), "agrião real" (Centranthus calcitrapae), "arruda" (Ruta chalepensis), "carqueja" (Pterospartum tridentatum), "carrasco" (Quercus ilex subsp. ballota), "cheirosinha" (Thymus zygis), "espinheiro" (Prunus spinosa), "erva-prata" (Paronychia argentea), "fiolho" (Foeniculum vulgare.), "tremoceiro bravo" (Lupinus angustifolius).

${ }^{9}$ También se ha realizado una categorización libre de las plantas ("free pilesort") que no será abordada en este texto.
} 


\section{perifèria}

Número 7, Diciembre 2007

www. periferia. name

La ruda (Ruta chalepensis), localmente conocida por "arruda" o "erva-da-inveja" ha sido nombrada por nueve sujetos de ambos sexos. Sin embargo, el número de los que le otorgaron una función protectora por su capacidad para ahuyentar las brujas y el mal de ojo es superior (12 informantes), pese a que algunos ( 6 personas) no conozcan su nombre vulgar. Es decir, parte de los informantes que conocía su valor simbólico no sabía su nombre. La mayoría (10 sujetos) de los conocedores del interés de la ruda son mujeres, 8 adultas, una joven de 16 y una niña de 11 años. Resaltamos que los dos varones pertenecientes al grupo de conocedores de ruda tienen historias de vida excepcionales. Se trata de un joven cuya familia es adepta y recurre con frecuencia a las terapias alternativas y de un varón casado con una mujer originaria de Brasil, país donde la planta tiene una simbología similar. Así sendo, podemos presumir que el conocimiento de la ruda nos remite para el carácter femenino del manejo ritual de las plantas en los contextos rurales portugueses.

En síntesis, el análisis preliminar y cualitativo de las "entrevistas con plantas" nos permite enumerar las siguientes tendencias: (i) los individuos más jóvenes, con edades inferiores a 40 años, desconocen tanto la nomenclatura local como la libresca y presentan dificultades a la hora de reconocer las plantas; (ii) aparentemente no hay diferencias en el conocimiento de sujetos mayores con distintos niveles de escolarización; (iii) los usos medicinales de las plantas son reconocidos por un conjunto de individuos con más edad, que no son necesariamente personas mayores, pero son varones y mujeres localmente muy considerados por su sabiduría acerca de plantas que hemos designados por expertos (especialistas) locales.

\section{El conocimiento acerca de plantas en la "nueva ruralidad"}

Al parecer hay dos sistemas de fuerzas distintos en esta "nueva ruralidad". Por una parte está la búsqueda de una confirmación erudita de la importancia de los recursos vegetales, llevada a cabo por los expertos locales, que crea una dinámica de enriquecimiento del patrimonio regional, además de un modo de se comunicar y identificar con valores que son entendidos como globales. Por otra, el desinterés de los más jóvenes que parece conducir a una perdida de la memoria cultural, porque 


\section{perifèria}

Número 7, Diciembre 2007

www. periferia.name

aunque viviendo en un medio rural, estos jóvenes han estado siempre apartados de las actividades agrarias y de los conocimientos etnobotánicos tradicionales.

Los conocimientos etnobotánicos tradicionales compartidos en Quintanilha coexisten con saberes exógenos. Al mismo tiempo que ocurre una pérdida de la importancia de muchas prácticas sociales con recurso a las plantas, asistimos a una separación entre el saber sobre plantas y el saber hacer con plantas, que propicia un espacio para la imposición de los conocimientos librescos. Además de otros factores concomitantes, como sean las políticas agrarias y de desarrollo rural, esta dualidad de aptitudes, entre el saber y el saber hacer, también interfiere con la gestión y manutención de los sistemas agrarios tradicionales ( $y$ también de los agroecosistemas) basada en prácticas, individuales o comunales y en relaciones jerárquicas ancestrales. En los pueblos apenas hay gente que sabe meter mano a la labor de gestionar el uso del agua o de las leñas, de manejar los pastos, bosques y tierras comunitarias o de dirigir la recría colectiva del ganado.

Estos sucesos afectan fundamentalmente los territorios de espacios naturales protegidos, debido a que su estatuto de protección está dependiente de la capacidad de mantener los niveles de biodiversidad y de conservación de los recursos naturales.

Sobre todo en los territorios donde la riqueza y diversidad de los ecosistemas van asociadas a una presencia humana, instalada desde tiempos inmemoriales, (que ha sabido intervenir de forma más o menos sostenible y conformar el paisaje y todo el territorio), la erosión de los conocimientos sobre flora y fauna, así como, el abandono de ciertas actividades agrícolas (claramente relacionadas con el manejo del universo vegetal) conducirá a cambios profundos en la estructura ecológica y social de estas áreas. En conclusión, considerando que la conservación no se limita al patrimonio biológico sino que incluye el paisaje, el manejo de los ecosistemas y de las plantas cultivadas y la integración del hombre en el ecosistema (Pardo de Santayana \& Gómez 2003), en la "nueva ruralidad", la erosión de los conocimientos y el abandono de las actividades agrarias produce cambios apreciables que afectan los presupuestos básicos que han dado origen al estatuto de espacio protegido, porque ocurre, en simultaneo, una erosión del patrimonio natural y del cultural, que 


\section{perifèria}

Número 7, Diciembre 2007

www.periferia. name

son indisociables. Además, la preservación del conocimiento y de las prácticas tradicionales tiende a confundirse con actitudes patrimonialistas o con una cristalización de memorias que non son incorporadas o evocadas en las prácticas sociales, principalmente por los más jóvenes;

Al límite y desde un punto de vista muy estricto, es como si el mundo rural se convirtiera en un club recreativo para turistas o ciudadanos de grandes urbes y parte de los residentes en esas áreas fueran tan solo los "jardineros del paisaje" y los guardianes de saberes que van perdiendo su sentido cultural, como nos indica Mormon (1987: 4):

Transforming agricultural areas in this way is seen as the last stage of a process - set in motion at least three decades ago - converting productive land for the recreation of city-dwellers, and where a few peasants of historical interest would remain primarily for urban use, as 'gardeners of the countryside'.

\section{Bibliografía}

Baptista, F. de Oliveira (1993). Agricultura, espaço e sociedade rural. Coimbra: Fora do Texto.

Brito, J. Pais de, Baptista, F. O. y Pereira, B. (coord.) (1996). O voo do arado. Lisboa: Museu Nacional de Etnologia, Instituto Português de Museus, Ministério da Cultura.

Carvalho, A. M. (2005). Etnobotánica del Parque natural de Montesinho. Plantas, tradición y saber popular en un territorio del nordeste de Portugal. [Tesis doctoral en Biología Evolutiva y Biodiversidad. Facultad de Ciencias]. Madrid: Universidad Autónoma de Madrid.

Ellen, Roy (2000). "Introduction" en Ellen, R., Parkes, P y Bicker, A. (eds.) Indigenous Environmental Knowledge and its Transformations. Critical Anthropological Perspectives. Amsterdam: Harwood Academic Publushers, pp. 1-33. ESAB/PNM (Escola Superior Agrária de Bragança/Parque Natural de Montesinho) (2007). Plano de Ordenamento do Parque Natural de Montesinho. Bragança: ESAB. 


\section{perifèria}

Número 7, Diciembre 2007

www.periferia.name

Godinho, Paula (2006). O Leito e a Margem. Estratégias familiares de renovação e situações liminares em seis aldeias do Alto Trás-os-Montes raiano. Lisboa: Colibri.

Milton, Kay (1996). Environnementalism. The View from Anthropology. London: Routledge.

Mormont, Marc (1987). "Rural Nature and Urban Nature". Sociologia Ruralis, vol. XXVII-1: 3-20.

O'Neill, Brian J. (1984). Proprietários, Lavradores e Jornaleiros. Lisboa: Dom Quixote.

Pardo de Santayana, M. y Gómez Péllon, E. (2003). “Etnobotánica: aprovechamiento tradicional de plantas y patrimonio cultural". Anales Jardín Botánico de Madrid 60 (1):171-181.

Pina-Cabral, João (1986). Filhos de Adão, Filhas de Eva. A Visão do Mundo Camponesa no Alto Minho, Lisboa: Dom Quixote.

Pinto, J. Madureira (1985). Estruturas Sociais e Práticas Simbólico-Ideológicas nos Campos. Elementos de Teoria e de Pesquisa Empírica. Porto: Ed. Afrontamento.

Rodrigues, Orlando I. A. (1998). A terra e a mudança. Reprodução Social e património fundiário na Terra Fria Transmontana. Bragança: Série Estudos, Instituto Politécnico de Bragança. 


\section{perifèria}

Número 7, Diciembre 2007

www.periferia.name

\section{Apéndice}

\begin{tabular}{|c|c|c|}
\hline NOMBRE POPULAR & NOMBRE CI ENTÍ FI CO & FAMI LI A BOTÁNI CA \\
\hline Abrunhos & Prunus spinosa L. & ROSACEAE \\
\hline Aarião & Rorippa-nasturtium- aquaticum (L.) Havek & BRASSICACEAE \\
\hline Aarião-real & Centranthus calcitrapae (L.) Dufr. & GENCIANACEAE \\
\hline Alcária, erva alcária & Xolantha tuberaria (I.) Galleqo Muñoz \& C. Navarro & CISTACEAE \\
\hline Amieiro & Alnus alutinosa (L.) Gaertn. & BETULACEAE \\
\hline Arcã, arcenha, rosmaninho & Lavandula stoechas L. & LAMIACEAE \\
\hline Arruda, erva da inveia & Ruta chalepensis L. & RUTACEAE \\
\hline Azedas & Rumex acetosa L. & POLYGONACEAE \\
\hline Azedas & Rumex induratus Boiss. \& Reut. & POLYGONACEAE \\
\hline Azedões. labaca & Rumex obtusifolius L. & POLYGONACEAE \\
\hline Borragem & Boraqo officinalis L. & BORAGINACEAE \\
\hline Botas & Hipochoeris alabra L. & ASTERACEAE \\
\hline Canastras & Astraqallus pelecinus (L.) Barnebv & FABACEAE \\
\hline Caneleiro. sabuaueiro & Sambucus niara L. & CAPRIFOLIACEAE \\
\hline Carneixos, cornicós & Astraqalus cvmbaecarpos Brot. & FABACEAE \\
\hline Carqueia & Pterospartum tridentatum subsp. tridentatum (L.) Willk. & FABACEAE \\
\hline Carrasco & Ouercus ilex subsb. ballota (Desf.) Samn. & FAGACEAE \\
\hline Castanheiro & Castanea sativa Mill. & FAGACEAE \\
\hline Cerdeiro, cereieira & Prunus avium L. & ROSACEAE \\
\hline Conecos, coneios & Silene vulaaris (Moench) Garcke & CARYOPHYLLACEAE \\
\hline Cheirosinha & Thvmus zvais L. & LAMI ACEAE \\
\hline Choupo híbrido & Populusxcanadensis Moench & SALICACEAE \\
\hline Choupo nearo & Populus niara L. & SALICACEAE \\
\hline Chupetas & Trifolium pratense L. & FABACEAE \\
\hline Erva prata & Paronvchia araentea Lam. & CARYOPHYLLACEAE \\
\hline Erva da tensão, urtiqa neara & Urtica dioica L. & URTICACEAE \\
\hline Escova amarela, aiesta & Cvtisus scoparius (L) Link & FABACEAE \\
\hline Esteva & Cistus ladanifer L. & CISTACEAE \\
\hline Fiolho, funcho & Foeniculum vulaare Mill. & UMBELLIFERAE \\
\hline Foqacas & Malva svlvestris L. & MALVACEAE \\
\hline Gilbardeira, aibardeira & Ruscus aculeatus L. & LI LIACEAE \\
\hline Gravanceira, arabanceira & Rosa canina L. & ROSACEAE \\
\hline Lentisco & Phvllvrea anqustifolia L. & OLEACEAE \\
\hline Pelicos, erva peliceira & Arctium minus (Hill) Bernh. & ASTERACEAE \\
\hline Rabacão & Apium nodiflorum (L.) Laq.. & UMBELLIFERAE \\
\hline Riaueiiões & Conopodium maius (Gouan) Loret & UMBELLIFERAE \\
\hline Salqueiras, salqueiro, veraas & Salix atrocinerea Brot, Salix salviifolia Brot & SALICACEAE \\
\hline Tremoceiro bravo & Lupinus anqustifolius L. & FABACEAE \\
\hline Urze roxa & Erica australis L. & ERICACEAE \\
\hline Vimes & Salix rubens Schrank & SALICACEAE \\
\hline Violeta & Viola riviniana Reichenb.. & VIOLACEAE \\
\hline Violeta branca & Viola odorata L. & VIOLACEAE \\
\hline
\end{tabular}

Tabla 1. Lista de las especies citadas en el texto: nomenclatura popular y científica y familia botánica. 


\section{perifèria}

Número 7, Diciembre 2007

www.periferia.name 